\title{
Actualización: Evolución y transformación conceptual de la Atención Primaria de la Salud
}

Evolution and conceptual transformation of Primary Health Care

Mariela Weisbrot

\begin{abstract}
Resumen
La Atención Primaria de la Salud (APS) ha generado una enorme cantidad de debates, que han girado en torno a las posibilidades reales de su implementación y en función de las transformaciones de su significado.

La estrategia APS comenzó siendo contradictoria en lo conceptual y fue atravesada por todas las transformaciones sufridas por nuestra sociedad durante los últimos 30 años.

Si bien la ambigüedad de sus postulados iniciales y la magnitud de los objetivos propuestos contribuyeron a la adhesión de una enorme cantidad de países sumamente heterogéneos entre sí, con el tiempo, esta misma ambigüedad fue volviéndose fuente de enormes discusiones. El objetivo de este artículo es describir su evolución y su transformación conceptual.
\end{abstract}

\section{Abstract}

Primary Health Care (PHC) has generated a huge amount of debate about the real possibilities of its implementation and in terms of the transformations of its meaning. PHC strategy began conceptually contradictory and was crossed by all the transformations of our society over the past 30 years.

The ambiguity of its initial assumptions and the magnitude of its objectives contributed to the adhesion of a huge amount of very heterogeneous countries, but over time, this ambiguity became a source of great discussions. The aim of this article is to describe its evolution and its conceptual transformation.

Palabras clave: atención primaria de la salud, funciones, reforma. Key words: primary health care, functions, reform.

Weisbrot. Evolución y transformación conceptual de la atención primaria de la salud. Evid Act Pract Ambul. 2014:17(3).Jul-Sep. 100-103.

\section{Introducción}

Las características y los objetivos de la Atención Primaria de la Salud' (APS) se plantearon hace más de tres décadas durante la conferencia de Alma Ata ${ }^{2 \varsigma}$.

Desde entonces y en pos de garantizar el derecho universal a la salud, esta estrategia se transformó en la política con mayor nivel de consenso a nivel global entre los actores del sector. Sin embargo, a la hora de ser implementada, este consenso se diluye y aparecen diferencias sustanciales.

Más allá de las particularidades nacionales, esta propuesta surgió y fue aceptada universalmente en función de un diagnóstico global que alertaba sobre la gran desigualdad existente en la condición de salud de las personas, especialmente entre los países desarrollados y los países en desarrollo, pero también dentro de cada nación ${ }^{2}$.

Así, a partir del conocimiento universal de la salud como derecho y como objetivo social, y en el convencimiento de que a la misma debe llegarse de forma contextualizada, los organismos sanitarios internacionales propusieron la estrategia de la APS como instrumento idóneo para transformar los sistemas de salud y para mejorar las condiciones de salud general.

Sin embargo, el pasaje desde la formulación teórico-metodológica hacia la implementación política de la APS no ha sido sencillo. La falta de discusión política, los costos excesivos, la dificultad para producir cambios institucionales y la imposibilidad de disciplinar a todos los actores del sector salud en torno a un sistema único articulado a través de una estrategia única, fueron algunos de los factores que obstaculizaron, particularmente en América Latina, la puesta en marcha de una APS integral como lo propiciaban la Organización Panamericana de la Salud (OPS) y la OMS³
A esto debe sumarse que a partir de los inicios de la década del "ochenta" comenzó a hacerse dominante en el mundo la perspectiva neoliberal en relación al rol del Estado y sus políticas sociales. En este contexto, pensar la política de salud en términos de sistemas nacionales, en los que el Estado asuma un rol preponderante y cuyas políticas de carácter universal tiendan a alcanzar una cobertura del $100 \%$ de la población como lo propone la estrategia de la APS, se transformó en una visión de política sanitaria carente de consenso social y político.

Desde este marco se desarrolló un intenso debate en torno a la supuesta inviabilidad política de la estrategia de APS y a la necesidad de su reconceptualización, lo que condujo a que el término APS dejara de tener un único significado.

Finalmente, durante los años "noventa" predominó en el diseño y en la ejecución de las políticas sociales un modelo minimalista en lo social. Respecto de las políticas de salud, este modelo significó la definición de una canasta básica de prestaciones y la orientación de los recursos del Estado solamente hacia los grupos más desfavorecidos $^{\star *}$. La APS pasó a ser sinónimo de programas sanitarios compensatorios o de emergencia destinados a los llamados "grupos vulnerables" de la población, transformándose en una herramienta central para los programas de atención de la pobreza extremat".

En la actualidad y bajo el mismo rótulo, se esconden diversas maneras de entender la APS: como una estrategia, como un nivel de atención y como un programa específico ${ }^{4}$. Así, programas de APS absolutamente aislados del resto del sistema de salud y sin conexión alguna con la red sanitaria pública, convierten a esta estrategia en una atención médica de segunda categoría. Dicho de otro modo y como Sostiene Testa ${ }^{5}$, la atención primaria de la salud se fue convirtiendo en "atención primitiva" de la salud.

‡ Servicio de Medicina Familiar y Comunitaria del Hospital Italiano de Buenos Aires y Departamento de Salud Pública del Instituto Universitario Hospital Italiano de Buenos Aires. mariela.weisbrot@ hiba.org.ar

$\S^{\S}$ En el transcurso de la Asamblea General de la Organización Mundial de la Salud (OMS) de 1977 se planteó la necesidad de garantizar la "Salud para todos en el año 2000". Por eso, durante 1978, en la reunión llevada a cabo en Alma Ata, quedó establecido que para el logro de esta meta era necesaria una estrategia específica, distinta a las utilizadas hasta entonces, que se definió como Atención Primaria de la Salud (APS) y a la que adhirió el Estado argentino.

** Los organismos financieros internacionales, en particular el Banco Interamericano de Desarrollo y el Banco Mundial, promocionaron a la APS como una estrategia de reforma y fortalecimiento de los sistemas de salud regionales en un sentido claramente selectivo.

"La APS se convirtió en un ejemplo claro de un modelo de política social, en donde ésta se limita a cumplir la función de "ambulancia" que recoge los heridos que va dejando la política económica. 


\section{¿Qué es la Atención Primaria de la Salud?}

La $\mathrm{APS}^{6}$ es la estrategia mediante la cual el sistema de salud procura influir sobre los determinantes de la salud de la población a través de un trabajo intersectorial y de la coordinación de acciones entre los distintos niveles de atención del sistema de salud ${ }^{\ddagger}$.

Se define como APS a aquel nivel del sistema sanitario accesible a todas las personas a lo largo del tiempo. Está centrado en la persona más que en la enfermedad, ofrece atención a todos o a la mayoría de sus problemas de salud (con excepción de los menos frecuentes) y coordina en forma integrada la atención prestada en otros lugares o por otros profesionales. Es un enfoque que constituye la base y determina la manera de trabajar de los restantes niveles del sistema sanitario $\$$.

La APS también implica el manejo de pacientes con múltiples enfermedades y cuadros complejos no clasificados entre los diagnósticos habituales. Podemos decir que una atención médica eficaz no se limita al tratamiento de la enfermedad, sino que también debe tener en cuenta el entorno en el que aparece dicho problema de salud y en el que vive la persona que la sufre, es decir su contexto***

En el marco de la estrategia de la APS, el sistema de salud tiene la responsabilidad de garantizar una cobertura y un acceso universal a servicios de salud de calidad, aceptables para la población. Al interior del sistema de salud, los servicios de primer nivel de atención tienen la responsabilidad de constituirse en la puerta de entrada al sistema (primer contacto) ${ }^{+1+5}$.

Un sistema de salud basado en la APS debería estar formado por un conjunto de elementos estructurales y funcionales esenciales que garanticen la cobertura y el acceso universal a servicios que sean aceptables para la población y promueven la equidad. Debería prestar atención integral, integrada y apropiada a lo largo del tiempo, poner énfasis en la prevención y la promoción, y garantizar el primer contacto del usuario con el sistema, tomando a las familias y a las comunidades como base para la planificación y la acción. Por lo tanto, se considera que una estrategia renovada de la APS es una condición esencial para lograr objetivos de desarrollo a nivel internacional, como los propuestos en la Declaración del Milenio (ODM), así como para abordar los determinantes fundamentales de la salud?

\section{Una definición renovada de la atención primaria de la salud}

La OMS define ${ }^{6}$ un sistema de salud basado en APS como un enfoque amplio de la organización y operación de los sistemas de salud que hace del derecho a alcanzar el mayor nivel de salud posi- ble su principal objetivo, al tiempo que maximiza la equidad y la solidaridad del sistema

La esencia de la definición renovada de la APS sigue siendo la misma que la de la Declaración de Alma Ata. Sin embargo, la nueva definición se centra en el sistema de salud en su conjunto; incluye a los sistemas público, privado y sin fines de lucro, y es aplicable a todos los países.

Distingue entre valores, principios y elementos, subraya la equidad y la solidaridad, e incorpora principios nuevos como la sustentabilidad y la orientación hacia la calidad. Especifica un grupo de elementos estructurales y funcionales que pueden medirse y evaluarse, y que constituyen una aproximación coherente y lógica hacia los sistemas de salud firmemente basados en la estrategia de APS $\$ s$.

Reconoce que la APS es algo más que la prestación de servicios de salud en el sentido estricto y que su éxito depende de otras funciones del sistema de salud y de otros procesos sociales.

Los servicios orientados hacia la calidad son aquellos que, además de responder a las necesidades de la población, tienen la capacidad de anticiparse a ellas y de tratar a todas las personas con dignidad y respeto, al tiempo que aseguran la mejor intervención para sus problemas de salud, minimizando la probabilidad de que ocurra cualquier daño. El Estado es el responsable último de garantizar la equidad y la calidad de la atención de la salud. Por consiguiente, la rendición de cuentas exige el monitoreo y la mejora continua del desempeño del sistema de salud de forma trasparente y sujeta al control social.

\section{Funciones de la Atención Primaria de la Salud}

Bárbara Starfield ${ }^{8}$ organizó un modelo conceptual para definir la APS que incluye cuatro atributos o funciones principales de la APS que se pueden organizar en subdimensiones estructurales y de procesos de los sistemas o servicios de salud, y tres funciones secundarias.

Esta definición clara y precisa de las funciones de la APS, permitió desarrollar una herramienta para evaluar el grado de implementación de la APS: Primary Care Assessment Tools: PCAT) ${ }^{9}$.

\section{Funciones esenciales de la Atención Primaria de la Salud}

Las cuatro funciones esenciales de la APS son?: 1) el primer contacto, 2) la continuidad, 3) la coordinación, 4) la integralidad o globalidad.

\footnotetext{
${ }^{\$ \varsigma}$ La estrategia APS aborda los problemas más frecuentes de la comunidad proporcionando servicios preventivos, curativos y de rehabilitación capaces de maximizar la salud y el bienestar de las personas. Integra la atención cuando existe más de un problema de salud, se enfrenta a los condicionantes de la enfermedad e influye en las respuestas de las personas a sus problemas de salud.

*** Si bien la especialización contribuye a la atención de personas con enfermedades específicas, es poco probable pueda brindar una atención básica de la salud que sea efectiva, ya que las actividades de prevención y promoción de la salud van más allá de las enfermedades específicas y su abordaje requiere una perspectiva más amplia que la del especialista. Un sistema sanitario centrado en la súper especialización presenta, además, el inconveniente de poner en peligro la equidad, ya que no existe ninguna sociedad que disponga de recursos económicos ilimitados para costear los servicios de salud.

${ }^{t+t} \mathrm{Al}$ interior de los servicios de primer nivel se constituyen equipos de trabajo, preferentemente multidisciplinarios. Los equipos tienen la responsabilidad -no exclusiva- de prestar atención integral, integrada y apropiada a las características de su población. Los equipos de salud proveen a su población atención por todos sus problemas de salud -exeptuando las más inusuales o las que requieren de alguna conocimiento técnico poco difundido- en coordinación con el resto del sistema, desarrollan acciones de prevención y promoción de la salud, promueven la participación comunitaria y coordinan acciones con otros sectores u organizaciones que tienen influencia sobre los determinantes de la salud. En este sentido, las familias y las comunidades son la base para la planificación y la acción.

\#\# Un sistema de tal naturaleza se guía por los principios propios de la APS que pretenden dar respuesta a las necesidades de salud de la población con participación intersectorial. Debe ser sustentable y estar orientado hacia la calidad, e incluir rendición de cuentas de los gobiernos.

${ }^{s \varsigma s}$ Está destinada a guiar las transformaciones de los sistemas de salud de manera que puedan alcanzar sus objetivos y ser los suficientemente fle-
} xibles como para adaptarse a los nuevos desafíos que vayan surgiendo con el transcurso del tiempo. 
El primer contacto supone la idea de que cada vez que surge un problema nuevo de salud se acude a un centro o profesional concreto, que actúa como puerta de entrada al sistema sanitario y es accesible a la población. Esta función se compone de dos subdimensiones: la accesibilidad y la continuidad.

La accesibilidad implica la existencia del elemento estructural necesario para el primer contacto y la utilización de los servicios ofrecidos como primer contacto (si el centro o profesional proveedor de APS es realmente visitado ante un problema o evento nuevo).

La continuidad observa la relación personal a largo plazo entre el usuario y el profesional o centro de salud, que debe facilitar el desarrollo de una relación basada en la confianza y en el conocimiento de la persona y su familia. Esta función se compone de dos subdimensiones: el grado de afiliación (medida en la que los usuarios identifican una fuente habitual de atención de la salud y en la que los centros de atención son capaces de identificar la población elegible o candidata a sus servicios) y la continuidad interpersonal (atención longitudinal focalizada en la persona y no en la enfermedad).

La coordinación es la función de enlace entre los servicios de atención, de tal modo que la persona reciba un cuidado apropiado de todos sus problemas de salud bajo la responsabilidad de su proveedor de APS. Permite el reconocimiento de problemas anteriores y nuevos e incluye también la referencia y el acompañamiento de la atención realizada en otros servicios especializados. La coordinación se compone de dos subdimensiones: el sistema de información (disponibilidad de instrumentos de registro, incorporación de esa información al plan de atención de la persona y desarrollo e implementación de un plan apropiado de identificación y gestión de necesidades de la población) y la integración de la información (mecanismos de transferencia de la información y de recepción de otras fuentes que pueden estar involucradas en la atención de las personas).

Por último, la integralidad o globalidad, se refiere a la característica de la organización que pueda ofrecer un catálogo extenso, integral y en forma articulada de todos los servicios que la población necesita. Incluye la identificación de problemas de todo tipo, sean orgánicos, funcionales o sociales, particularmente aquellos más frecuentes en la población.

Esta dimensión se evalúa respecto de los servicios disponibles (aquellos servicios con los que el centro cuenta para ser provistos cuando sean necesarios) y de los servicios proporcionados (los servicios necesarios para la población, que el centro realmente provee).

\section{Funciones secundarias de la Atención Primaria de la Salud}

Por otro lado, las tres funciones secundarias de la APS son el enfoque familiar, la orientación comunitaria y la competencia cultural. El enfoque familiar se refiere a la característica de centrar la atención de la salud de los individuos en su contexto social más cercano. La orientación comunitaria se refiere a la elaboración de programas de atención basados en el conocimiento de las necesidades particulares de una población definida y la competencia cultural describe si el centro adapta y establece relaciones que faciliten la atención de los problemas de salud en las personas de diferentes grupos sociales de la población asignada.

\section{Atención Primaria de la Salud en Argentina ${ }^{10}$}

La APS no es ni ha sido una política prioritaria para la salud pública en Argentina ya que el modelo de atención ha sido histórica- mente "hospitalocéntrico".

La declaración del Alma Ata de 1978 encontró a nuestro país transitando un proceso que había comenzado dos años antes, el 24 de marzo del 1976, con una nueva interrupción de un gobierno democrático por las fuerzas armadas, con la instalación de una política sistematizada de desaparición de personas, calificación de las ideologías progresistas como enemigas de la Nación y la prohibición expresa de cualquier participación política. Bajo este modelo, la Salud pública dejó de plantearse como responsabilidad del Estado a través de medidas como la transferencia de efectores a las provincias, sin el respaldo de los recursos.

La reducción de la capacidad orientadora y fiscalizadora del Estado, generó el desarrollo de un mercado de servicios de salud, en el que el uso indiscriminado e innecesario de recursos tecnológicos pasó a ser la constante, consolidándose un modelo de servicios de alta especialización que privilegió la atención de determinadas patologías en detrimento de acciones basadas en la prevención y la promoción de la salud ${ }^{3}$. En este contexto, la incorporación de la APS en Argentina fue expresamente restringida a algunas dimensiones técnicas, aplicadas en las zonas libres de conflicto. Siempre con una expresa supresión de sus componentes más políticos, como la participación comunitaria y la intersectorialidad.

Durante la década de los noventa, Argentina se sumó a la adopción de políticas neoliberales que vaciaron el Estado y a la aparición del mercado como la única voz autorizada para dictar la agenda pública. La desvalorización del Estado intervencionista, las estrategias de privatización, descentralización y focalización en el intento de establecer un orden, impactaron con especial dureza en la situación de la salud, la nutrición y la educación de crecientes sectores sociales (prescripciones del denominado "Consenso de Washington") $)^{10}$.

Respecto a las políticas sanitarias, se consolidó en Argentina una fuerte tendencia hacia la mercantilización de la salud, expresada a través de la heterogeneidad en la calidad de distintas prácticas. Por un lado, servicios de alta calidad con costos que sólo unos pocos podrían pagar y por el otro, una oferta de servicios, ostensiblemente de menor calidad, destinados a sectores populares, quedando bien delimitada dicha frontera .

En este contexto, la estrategia de la APS se vio traducida como el acceso a un paquete mínimo de intervenciones focalizadas que ocultaba los determinantes sociales, económicos, culturales y urbanísticos, y lógicamente incapaz de dar respuesta al desafío que implicaba generar un nuevo modelo de atención intercultural para abordar la cuestión migratoria.

Luego de la crisis del año 2001 comenzó un nuevo período caracterizado por cambios "acompasados" en la región latinoamericana y particularmente del cono sur; con gobiernos progresistas que apuestan a la importancia geopolítica de la región, a cierta redistribución del ingreso y al resurgimiento de Estados presentes, que aparecen como reguladores en pro del bienestar de la ciudadanía. En este escenario se reinstala lentamente el concepto de "Atención Primaria de la Salud" como políticamente correcto, sin que logre reflejarse en políticas transformadoras que apunten, en la práctica, a resultados de equidad en salud.

\section{A modo de conclusión}

Entendemos que unas de las principales debilidades de la estrategia de APS en Argentina es que no estuvo formulada en su origen, en términos estratégicos, tanto en la formación de los recursos humanos como en la práctica cotidiana. A lo largo del tiempo, en la política sanitaria operaron dispositivos de represión, disciplinamien- 
to y orientación de las prácticas de APS, delimitando a priori "en qué se debe meter y en qué no" el personal de salud que esta en el Primer Nivel de Atención ${ }^{\star \star \star \star}$.

Otros obstáculos igualmente importantes que inciden para la efectivización plena de APS Integral en nuestro país son el poder de la corporación médica, la industria farmacéutica y de las tecnologías de diagnóstico, así como también la falta de planificación en el primer nivel de atención en base a las características poblacionales, la cristalización de modelos formativos que no dan respuesta a las principales problemáticas de salud de los conjuntos sociales y el desinterés de los profesionales que intervienen en el primer nivel de atención, probablemente consecutivo a su baja jerarquización en términos salariales y/o de crecimiento y prestigio laboral.
Desde la declaración de Alma Ata han sido muchas las resistencias y los obstáculos, lo que se ha traducido en resultados muy disímiles entre los diferentes países y en que la meta "Salud para todos en el año 2000" siga siendo tan lejana como entonces. Respecto de Argentina en particular, podemos decir que todavía nos queda un gran camino para recorrer y que el resultado global de ese recorrido depende mucho más de la calidad institucional que vayamos logrando como nación ya que el Sistema de Salud y sus resultados no son ajenos del funcionamiento global del Estado y de nuestro compromiso como ciudadanos.

Recibido el 01/06/14 y aceptado el 03/08/14

\section{Referencias:}

1.Ase I y col. La estrategia de Atención Primaria de la Salud: ¿progresividad o regresividad en el derecho a la salud? Salud Colectiva, Buenos Aires ,2009: 5(1):27-47 2. WHO. Declaration of Alma-Ata, 1978. Disponible en: http://www.who.int/hpr/NPH/docs/declaration_almaata.pdf [Acceso 01/01/2014]

3. Ase I y col. La estrategia de Atención Primaria de la Salud: ¿progresividad o regresividad en el derecho a la salud? Salud Colectiva, Buenos Aires, 2009:5(1):27-47.

4. Bloch C. Atención Primaria de la Salud en Argentina, desarrollo y situación actual. (Mesa redonda). EN: Segundas jornadas de Atención Primaria de la Salud. Buenos Aires: Grupo Editor de las Jornadas; 1988. p75-90

5. Testa M. Atención ¿Primaria o Primitiva? de la salud. En: Segundas Jornadas de Atención Primaria de la Salud. Buenos Aires: Grupo Editor de las Jornadas; 1988. p. 75-90.

6. Haggerty $\mathrm{J}$ y col. Grupo de Consenso sobre un Marco de Evaluación de la Atención Primaria en América Latina. Un marco de evaluación de la atención primaria de salud en América Latina. Rev Panam Salud Publica. 2009;26(5):377-84

7. OPS/OMS. La Renovación de la Atención Primaria de la Salud en las Américas. Julio 2007.

8. Starfield B. Atención Primaria. Equilibrio entre necesidades de salud, servicios y tecnologías. Ed Masson, Barcelona, año 2001.

9. Shi L y col. Validating the Adult Primary Care Assessment Tool. J Fam Pract, 2001; 50(2):161.

10. Rovere M y col. La estrategia de APS en Argentina; crecimiento desordenado, componentes reprimidos y conflicto de modelos. Una reconstrucción a partir de testimonios, huellas y rastros en un estudio espacial y temporal. En: EL Agora. Buenos Aires, año 2011.

11. Wilner A y col. Abordajes de la Atención Primaria y el Derecho a la Salud en los países del Cono Sur - Red ISSS. Algunas ideas sobre la Atención Primaria de la Salud en Argentina. Mayo de 2007. Disponible en URL: http://salud.ciee.flacso.org.ar/files/flacso/Concepcion/pdf/anteriores/APS\%20y\%20Derecho.pdf

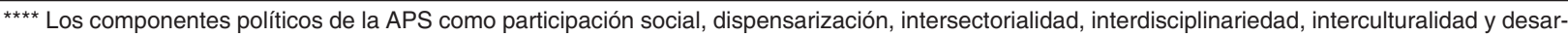
rollo de tecnologías apropiadas, no se encuentran o se encuentran muy acotados, normatizados y normalizados como para esterilizar o moderar su potencial de transformación. 